\title{
Soluble kagome Ising model in a magnetic field
}

\author{
W. T. $\mathrm{Lu}^{1,2}$ and F. Y. Wu ${ }^{1}$ \\ ${ }^{1}$ Department of Physics, ${ }^{2}$ Electronic Materials Research Institute, \\ Northeastern University, Boston, Massachusetts 02115
}

(Dated: November 10, 2018)

\begin{abstract}
An Ising model on the kagome lattice with super-exchange interactions is solved exactly under the presence of a nonzero external magnetic field. The model generalizes the super-exchange model introduced by Fisher in 1960 and is analyzed in light of a free-fermion model. We deduce the critical condition and present detailed analyses of its thermodynamic and magnetic properties. The system is found to exhibit a second-order transition with logarithmic singularities at criticality.
\end{abstract}

PACS numbers: $05.50 .+\mathrm{q}, 75.10 . \mathrm{HK}, 04.20 . \mathrm{Jb}, 51.60 .+\mathrm{a}$

\section{INTRODUCTION}

The Ising model in a nonzero magnetic field is a well-known unsolved problem in statistical mechanics. In 1960, Fisher [1] produced a remarkable solution of a super-exchange antiferromagnetic Ising model in the presence of a nonzero field. The Fisher model is defined on a decorated square lattice where there is an external magnetic field applied to decorating spins which interact via super-exchange interactions.

In this paper we consider similar super-exchange models on the kagome lattice. The kagome lattice has been of interest in recent years in the role it plays in the high- $T_{c}$ superconductivity. It has been known that special cases of the Kagome Ising model are soluble in the presence of a magnetic field [2, 3]. The structure of the ferrimagnet $\mathrm{SrCr}_{8} \mathrm{Ga}_{4} \mathrm{O}_{19}$, for example, is found to consist of $2 \mathrm{D}$ spinel (kagome) slabs 4] with magnetic spins residing at $1 / 3$ of the lattice sites. Thus it is of interest to consider models with similar structures. Azaria and Giacomini [5] have extended the Fisher model by considering the kagome lattice shown in Fig. 1(a), which reduces to the Fisher model upon setting $K_{1}=0$. They obtained its partition function from which the phase diagram is deduced. But there has been no detailed discussion of its thermodynamic properties. Here, we consider yet another extension of the Fisher model to a kagome lattice as shown in Fig. 1(b). We show that the partition function of this model is identical to the Azaria and Giacomini solution [5], although there appears to be no direct mapping between the two models. In addition, we also present detailed analyses of the thermodynamics of the solution.

Consider an Ising model shown in Fig. 1(b) with an interaction energy

$$
-J_{1} \sigma_{1} \sigma_{2}+J\left(\sigma_{2} \sigma_{3}-\sigma_{3} \sigma_{1}\right)
$$

around every triangle formed by spins $\sigma_{1}, \sigma_{2}, \sigma_{3}$. Introduce reduced interactions $K=\beta J, K_{1}=\beta J_{1}$, where $\beta=1 / k T$, such that $K, K_{1}>0$ indicate ferromagnetic interactions. In addition, there is an external magnetic field $H$ applied to $2 / 3$ of the lattice sites denoted by solid circles. We denote the reduced field by
$L=H / k T$. As a result, the magnetic spins interact with an super-exchange interaction via intermediate nonmagnetic spins. It is clear that there is no loss of generality to restrict considerations to

$$
H, J \geq 0, \quad \text { or } \quad L, K \geq 0
$$

For $K_{1}=0$ the model reduces to the Fisher model for the square lattice. For $K_{1} \neq 0$ the present model is more general and differs from the Fisher model in a fundamental way as described in Sec. 2 below.

Our main result is a closed-form solution of the partition function and detailed analyses of thermodynamic and magnetic properties. In Sec. 2 we deduce the solution using a combination of star-triangle and decimation transformations. The phase diagram is analyzed in Sec. 3. In Secs. 4 - 6 the internal energy, specific heat, magnetization, and susceptibility are analyzed using a free-fermion model formulation [6].

\section{THE PARTITION FUNCTION}

Denote the partition function of the kagome Ising lattice in Fig. $1\left(\right.$ b) by $Z_{\mathrm{KG}}\left(K, K_{1}, L\right)$. Our main result is an equivalence of $Z_{\mathrm{KG}}\left(K, K_{1}, L\right)$ to the partition function of an honeycomb Ising model in zero field, a result which renders the model soluble.

This equivalence is established by effecting a sequence of spin transformations. First, we carry out a startriangle transformation for every triangular face as shown in the first line in Fig. 2. This converts the kagome lattice to a decorated honeycomb lattice. Next the decorating spins are decimated as shown in the second and third lines in Fig. 2, and the lattice is reduced to that of an honeycomb. The crux of matter is that the external fields $L_{1}$ and $-L_{1}$ induced in the second step cancel out at the end. As a result, the final honeycomb lattice has no external field and is soluble. It has reduced interactions $R, R$ and $R_{1}$ in the three principal directions.

The transformations shown in Fig. 2 are standard 7 , 8]. For the transformation in the first line in Fig. 2, we 


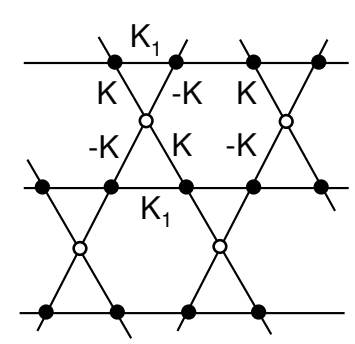

(a)

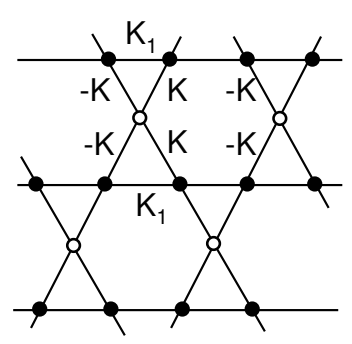

(b)

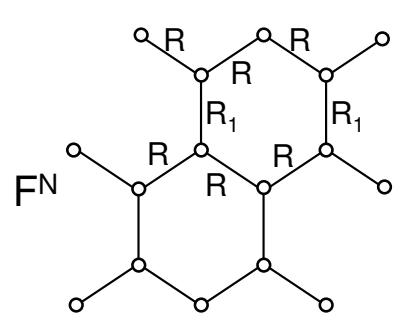

(c)

FIG. 1: (a) and (b) are two soluble Kagome lattice Ising models in a magnetic field. Solid circles denote magnetic spins and open circles non-magnetic spins. Both models are mapped to an honeycomb Ising lattice without a field shown in (c). $F^{N}$ is an overall factor.

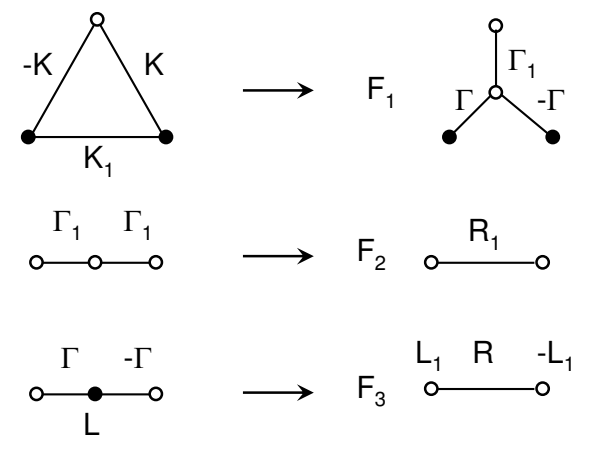

FIG. 2: Transformations used in deducing the mapping shown in Fig. 1. $F_{i}, i=1,2,3$ are constants.

have

$$
\begin{aligned}
e^{-2 K-K_{1}} & =2 F_{1} \cosh \left(\Gamma_{1}+2 \Gamma\right) \\
e^{2 K-K_{1}} & =2 F_{1} \cosh \left(\Gamma_{1}-2 \Gamma\right) \\
e^{K_{1}} & =2 F_{1} \cosh \Gamma_{1}
\end{aligned}
$$

from which we can solve $\Gamma$ and $\Gamma_{1}$ in terms of $K$ and $K_{1}$. Divide the sum and difference of (11) and (2) by (3), we obtain, respectively,

$$
\begin{aligned}
\cosh 2 \Gamma & =e^{-2 K_{1}} \cosh 2 K \\
\tanh \Gamma_{1} & =-\frac{e^{-2 K_{1}} \sinh 2 K}{\sinh 2 \Gamma} .
\end{aligned}
$$

We also obtain

$$
F_{1}^{4}=\left[16 \cosh ^{2} \Gamma_{1} \cosh \left(\Gamma_{1}+2 \Gamma\right) \cosh \left(\Gamma_{1}-2 \Gamma\right)\right]^{-1} .
$$

Now $K>0$ and $K_{1}$ real, so (4) and (5) show that there are two regimes

$$
\begin{array}{ll}
e^{-2 K_{1}} \cosh 2 K>1 & \text { regime I } \\
e^{-2 K_{1}} \cosh 2 K<1 & \text { regime II }
\end{array}
$$

and $\Gamma$ and $\Gamma_{1}$ are real in regime $I$ and pure imaginary in regime II.
For the transformation in the second line in Fig. 2, we have

$$
\begin{aligned}
2 \cosh 2 \Gamma_{1} & =F_{2} e^{R_{1}} \\
2 & =F_{2} e^{-R_{1}}
\end{aligned}
$$

from which we obtain

$$
\begin{aligned}
F_{2}^{2} & =4 \cosh 2 \Gamma_{1} \\
e^{2 R_{1}} & =\cosh 2 \Gamma_{1}=\frac{e^{-4 K_{1}} \cosh 4 K-1}{e^{-4 K_{1}}-1}
\end{aligned}
$$

where the last step is obtained after making use of (4). Equation (10) shows that $R_{1}$ is real, and $R_{1}>0$ in regime I and $R_{1}<0$ in regime II.

For the transformation in the third line in Fig. 2, we have

$$
\begin{aligned}
2 \cosh (L+2 \Gamma) & =F_{3} e^{-R+2 L_{1}} \\
2 \cosh (L-2 \Gamma) & =F_{3} e^{-R-2 L_{1}} \\
2 \cosh L & =F_{3} e^{R}
\end{aligned}
$$

from which we obtain

$$
\begin{aligned}
e^{-4 R} & =\frac{\cosh (L+2 \Gamma) \cosh (L-2 \Gamma)}{\cosh ^{2} L}=1+\frac{\sinh ^{2} 2 \Gamma}{\cosh ^{2} L} \\
& =\frac{e^{-4 K_{1} \cosh ^{2} 2 K+\sinh ^{2} L}}{\cosh ^{2} L} \\
e^{4 L_{1}} & =\frac{\cosh (L+2 \Gamma)}{\cosh (L-2 \Gamma)} \\
F_{3}^{4} & =16 \cosh ^{2} L \cosh (L+2 \Gamma) \cosh (L-2 \Gamma)
\end{aligned}
$$

Thus, $R$ is always real, and we have

$$
\begin{array}{ll}
R<0, R_{1}>0, & \Gamma, \Gamma_{1}, L_{1}=\text { real }, \quad \text { regime } \mathrm{I} \\
R>0, R_{1}<0, & \Gamma, \Gamma_{1}, L_{1}=\text { imaginery, regime } \mathrm{II}(17)
\end{array}
$$

This says that $R$ and $R_{1}$ have opposite signs. In the solution obtained by Azaria and Giacomini [5], the final equivalent honeycomb Ising model is identical with ours 
except the replacement of $R$ by $-R$. However, as we shall seen (23) below, the negations of $R$ and/or $R_{1}$ do not affect the solution. Therefore, the two solutions are identical.

Combining (6), (9), (16), and after some reduction, we obtain

$$
\begin{aligned}
F^{4} & =F_{1}^{4} F_{2}^{4} F_{3}^{2} \\
& =\frac{4 \cosh ^{2} L\left(e^{4 K_{1}} \sinh ^{2} L+\cosh ^{2} 2 K\right)}{\cosh ^{2} R_{1}} .
\end{aligned}
$$

Now the field $L_{1}$ at the decorating sites is canceled and we arrive at the final equivalence

$$
Z_{\mathrm{KG}}\left(K, K_{1}, L\right)=F^{N} Z_{\mathrm{HC}}\left[R(L), R_{1}\right]
$$

where $Z_{\mathrm{HC}}\left[R(L), R_{1}\right]$ is the partition function of the honeycomb Ising lattice. Here, $N$ is the number of triangular faces (or the number of magnetic spins) of the kagome lattice, which is also the number of honeycomb lattice sites. Note that the $L$ dependence is only in $R$.

The transformation (19) reduces to that of Fisher's for the square lattice after setting $K_{1}=0\left(R_{1}=\infty\right)$. But our model differs from the Fisher model in a crucial aspect: The Fisher model results in a square lattice whose sites are the decorating sites of the original lattice, in the present model all sites of the original kagome lattice disappear at the end. Thus the ordering of the honeycomb lattice bears no direct relationship to that of the kagome lattice. In addition, while there is no frustrated plaquettes in the Fisher model, in the $K_{1}>0$ model all triangular faces are frustrated.

Introduce the per magnetic spin free energy

$$
f_{\mathrm{KG}}\left(K, K_{1}, L\right)=\lim _{N \rightarrow \infty} N^{-1} \ln Z_{\mathrm{KG}}\left(K, K_{1}, L\right)
$$

and the per-site honeycomb lattice free energy

$$
f_{\mathrm{HC}}\left(R, R_{1}\right)=\lim _{N \rightarrow \infty} N^{-1} \ln Z_{\mathrm{HC}}\left(R, R_{1}\right) .
$$

Taking the $N \rightarrow \infty$ limit of (19) and making use of the explicit expression of $f_{\mathrm{HC}}$ given in [8, 9], we obtain for the kagome Ising model,

$$
\begin{aligned}
f_{\mathrm{KG}}\left(K, K_{1}, L\right)= & \ln F+f_{\mathrm{HC}}\left(R, R_{1}\right) \\
= & \ln F+\frac{3}{4} \ln 2 \\
& \quad+\frac{1}{16 \pi^{2}} \int_{0}^{2 \pi} d \theta d \phi \ln \Xi(\theta, \phi)
\end{aligned}
$$

where

$$
\begin{aligned}
\Xi(\theta, \phi)= & \cosh 2 R_{1} \cosh ^{2} 2 R+1-\sinh ^{2} 2 R \cos (\theta+\phi) \\
& -\sinh 2 R_{1} \sinh 2 R(\cos \theta+\cos \phi) .
\end{aligned}
$$

Note that the negation of either $R$ or $R_{1}$ corresponds to changing $\theta \rightarrow \pi-\theta, \phi \rightarrow \pi-\phi$ in (22) which does not change the free energy $f_{\mathrm{KG}}$.
As a check, we recover the Fisher solution [1] upon setting $K_{1}=0$ or $R_{1} \rightarrow \infty$ :

$$
\begin{aligned}
f_{\mathrm{KG}}(K, 0, L)=\frac{3}{2} \ln 2+\frac{1}{4} \ln \left[\cosh ^{2} L\left(\sinh ^{2} L+\cosh ^{2} 2 K\right)\right] \\
+\frac{1}{16 \pi^{2}} \int_{0}^{2 \pi} d \theta d \phi \ln \left[\cosh ^{2} 2 R\right. \\
\quad-\sinh 2 R(\cos \theta+\cos \phi)](23)
\end{aligned}
$$

with $e^{-4 R}=\left(\sinh ^{2} L+\cosh ^{2} 2 K\right) / \cosh ^{2} L$.

The internal energy and magnetization per magnetic spin are, respectively,

$$
\begin{aligned}
U\left(K, K_{1}, L\right) & =-\frac{\partial}{\partial \beta} f_{\mathrm{KG}}\left(K, K_{1}, L\right), \\
M\left(K, K_{1}, L\right) & =\frac{\partial}{\partial L} f_{\mathrm{KG}}\left(K, K_{1}, L\right) .
\end{aligned}
$$

The specific heat and susceptibility are further computed as

$$
\begin{aligned}
C_{H}\left(K, K_{1}, L\right) & =\frac{\partial}{\partial T} U\left(K, K_{1}, L\right), \\
\chi\left(K, K_{1}, L\right) & =\frac{\partial}{\partial H} M\left(K, K_{1}, L\right) .
\end{aligned}
$$

\section{THE PHASE DIAGRAM}

The integral in the free energy $f_{\mathrm{KG}}$ is precisely of the form of that of the free-fermion model discussed by Fan and $\mathrm{Wu}$ [6] for which the critical condition is (for $R R_{1}<$ $0)$

$$
\Xi(\pi, \pi)=0
$$

Alternately, the critical point for an anisotropic honeycomb Ising lattice has been given in [9] as $C_{1} C_{2} C_{3}+$ $1=S_{1} S_{2}+S_{2} S_{3}+S_{3} S_{1}$ where $C_{i}=\cosh 2 R_{i}, S_{i}=$ $\sinh \left|2 R_{i}\right|, i=1,2,3$. It is also given by the expression $\omega_{1}=\omega_{2}+\omega_{3}+\omega_{4}[6]$ where the $\omega$ 's are given in the next section. Using any of these expressions, we obtain the critical condition

$$
\sinh [2 R(L)]=-\operatorname{coth} R_{1}
$$

Explicitly, 29 reads

$$
\begin{aligned}
\cosh ^{2} L= & \frac{1}{2}\left[\sqrt{1+\tanh ^{2} R_{1}}-1\right] \\
& \times\left(e^{-4 K_{1}} \cosh ^{2} 2 K-1\right), \quad T=T_{c}(H(30)
\end{aligned}
$$

which can be realized only in regime I and $K_{1} \leq K$, or $\gamma \leq 1$ (see below). Note that for $K_{1}=0$ (30) reduces to the Fisher expression

$$
\cosh L=\sqrt{(\sqrt{2}-1) / 2} \sinh 2 K .
$$


It can be verified that we have

$$
|\sinh 2 R(L)|<\left|\operatorname{coth} R_{1}\right|, \quad T>T_{c}(H) .
$$

Introduce parameters

$$
\begin{aligned}
\alpha & =L / 2 K=H / 2 J>0 \\
\gamma & =J_{1} / J=K_{1} / K
\end{aligned}
$$

such that $\gamma>0$ indicates $J_{1}$ is ferromagnetic, and consider the phase diagram (30) in the $\{\alpha, 1 / K\}$ plane, where $1 / K$ is the temperature. The phase diagram is plotted in Fig. 3 for different fixed values of $\gamma$.

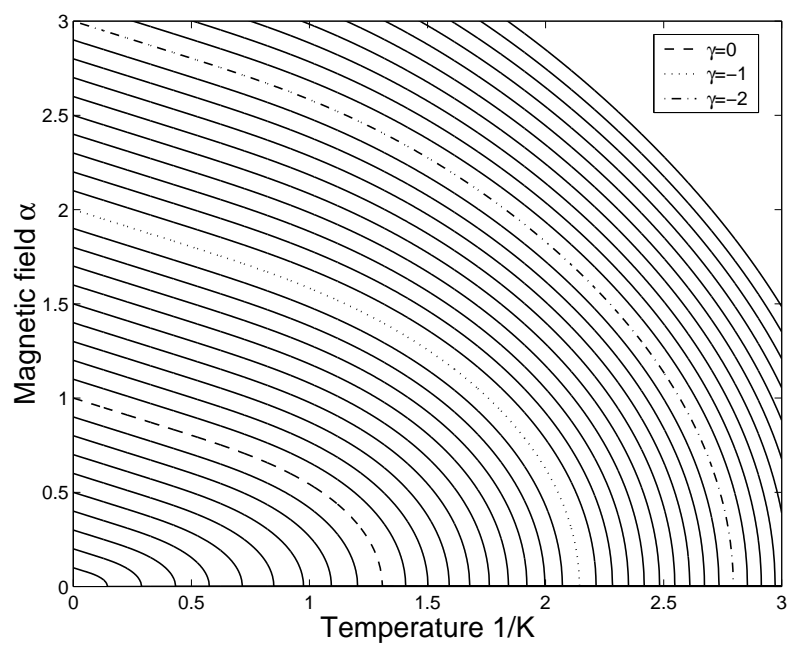

FIG. 3: Phase boundary in the $\alpha-T$ plane for fixed values of $\gamma$.

At low temperatures the phase boundary behaves as

$$
\alpha=1-\gamma-\frac{1}{4 K} \ln 2(\sqrt{2}+1)
$$

with an initial slope independent of $\gamma$. Solving (30) for $\gamma$ at $H=0$, we obtain

$$
\gamma=\frac{1}{2 K_{c}}\left[2 \ln \left(\sinh 2 K_{c}\right)-\ln \left(2 \cosh 2 K_{c}\right)\right] .
$$

We see that $T_{c}(0)$ decreases with $\gamma$, reaching $T_{c}(0)=0$ at $\gamma=1$. It follows that there is no transition when

$$
\gamma \geq 1, \quad \text { or } K_{1} \geq K
$$

as aforementioned. This can be understood physically since, in the limit of $\gamma \rightarrow \infty$, the non-magnetic spins can assume values \pm 1 randomly and there is no ordered state.

For small $H$ (30) is expanded as

$$
T_{c}(H)=T_{c}(0)-C H^{2},
$$

where $C$ is a constant given by

$$
C^{-1}=k J\left[\frac{32\left(16-24 t^{4}+t^{8}\right)}{t^{9}\left(4+4 t^{2}-t^{4}\right)}+\frac{16\left(-4 \gamma+4 t+t^{5}\right)}{16-t^{8}}\right]
$$

with $t=\tanh 2 J / k T_{c}(0)$.

To determine the nature of regimes in the phase diagram, we consider the ground state. At zero temperature the kagome lattice can assume two ordered states:

(i) All magnetic spins aligned in the same direction with the total energy

$$
U_{1}=-N(2 \alpha+\gamma) J
$$

independent of the spin directions of the non-magnetic spins. Since the $N / 2$ non-magnetic spins can point in any direction, the entropy of this ground state is $\frac{1}{2} N k \ln 2$.

(ii) The magnetic spins connected by interactions $-J$ assume the value +1 (or -1 ) and those connected by interactions $+J$ assume the value $-1($ or +1$)$, while all non-magnetic spins are fixed at +1 (or -1$)$. This forms a super-exchange antiferromagnetic state with the energy

$$
U_{2}=-N(2-\gamma) J
$$

and the ground state is two-fold degenerate with zero entropy in the thermodynamic limit.

Comparing $U_{1}$ with $U_{2}$, for $\alpha>1-\gamma$ which is the regime to the "exterior" of the critical curve (30), we have $U_{1}<U_{2}$ indicating the system is paramagnetic. For $\alpha<1-\gamma$ which is the regime enclosed by the critical curve, we have $U_{1}>U_{2}$ and the system assumes a superexchange antiferromagnetic ordering.

\section{THERMODYNAMIC PROPERTIES}

To analyze thermodynamic and magnetic properties given by (24) - (27), we make use of results of [6]. Writing (22) in the form of Eq. (16) of 6], we have

$$
\Xi(\theta, \phi)=2 a+2 b \cos \theta+2 c \cos \phi+2 d \cos (\theta+\phi),
$$

with

$$
\begin{aligned}
a & =\cosh 2 R_{1} \cosh ^{2} 2 R+1=\frac{1}{2}\left(\omega_{1}^{2}+\omega_{2}^{2}+\omega_{3}^{2}+\omega_{4}^{2}\right) \\
b & =-\sinh 2 R_{1} \sinh 2 R=\omega_{1} \omega_{3}-\omega_{2} \omega_{4} \\
c & =-\sinh 2 R_{1} \sinh 2 R=\omega_{1} \omega_{4}-\omega_{12} \omega_{3} \\
d & =-\sinh ^{2} 2 R=\omega_{3} \omega_{4}-\omega_{1} \omega_{2}
\end{aligned}
$$

or

$$
\begin{aligned}
& \omega_{1}=\cosh \left(2 R-R_{1}\right) \\
& \omega_{2}=\cosh \left(2 R+R_{1}\right) \\
& \omega_{3}=\omega_{4}=\cosh R_{1} .
\end{aligned}
$$

Using (42) we compute the parameters $\{x, y, z\}$ introduced in [6] as

$$
\begin{aligned}
& x=\omega_{1} \omega_{4}-\omega_{2} \omega_{3}=-\sinh 2 R \sinh 2 R_{1} \\
& y=\frac{1}{2}\left(\omega_{1}^{2}-\omega_{2}^{2}-\omega_{3}^{2}+\omega_{4}^{2}\right)=-\frac{1}{2} \sinh 4 R \sinh 2 R_{1} \\
& z=\omega_{1} \omega_{4}+\omega_{2} \omega_{3}=2 \cosh 2 R \cosh ^{2} R_{1} .
\end{aligned}
$$


Here we have reversed the sign of $x$ from [6] to make $x>$ 0 . This is permitted since only $x^{2}$ appears in the ensuing discussions. The critical condition (29) is equivalent to $y=z$ and we have the regimes

$$
\begin{array}{ll}
y>z>x>0, & T<T_{c}(H) \\
z>y>x>0, & T>T_{c}(H) .
\end{array}
$$

It was established in [6] that derivatives of the free energy are best computed by first carrying out one-fold integration. Adopting notations in [6], we obtain after differentiating (21) the expression

$$
\left[f_{\mathrm{KG}}\left(K, K_{1}, L\right)\right]^{\prime}=C_{0}+C_{1} I_{1}+C_{2} I_{2}+C_{3} I_{3},
$$

where the prime denotes the derivative with respective to some variable such as $T$ and $H$,

$$
\begin{aligned}
C_{0} & =\frac{F^{\prime}}{F}+\frac{b^{\prime}}{4 b}, \quad\left|R_{1}\right|>|R| \quad\left(b^{2}>d^{2}\right), \\
& =\frac{F^{\prime}}{F}+\frac{d^{\prime}}{4 d}, \quad\left|R_{1}\right|<|R| \quad\left(d^{2}>b^{2}\right), \\
C_{1} & =a^{\prime}-\frac{a}{2}\left(\frac{b^{\prime}}{b}+\frac{d^{\prime}}{d}\right)-\frac{b^{2}-d^{2}}{4 d}\left(\frac{b^{\prime}}{b}-\frac{d^{\prime}}{d}\right), \\
C_{2} & =\frac{b}{2}\left(\frac{b^{\prime}}{b}-\frac{d^{\prime}}{d}\right), \\
C_{3} & =-\frac{1}{2}\left(\frac{b^{\prime}}{b}-\frac{d^{\prime}}{d}\right) \frac{b^{2}-d^{2}}{2 d}\left(\frac{2 a d}{b^{2}+d^{2}}-1\right),
\end{aligned}
$$

and

$$
\begin{aligned}
& I_{1}=\frac{1}{8 \pi} \int_{0}^{2 \pi} d \phi[Q(\phi)]^{-1 / 2}, \\
& I_{2}=\frac{1}{8 \pi} \int_{0}^{2 \pi} d \phi \cos \phi[Q(\phi)]^{-1 / 2}, \\
& I_{3}=\frac{1}{8 \pi} \int_{0}^{2 \pi} d \phi(1+\omega \cos \phi)^{-1}[Q(\phi)]^{-1 / 2}
\end{aligned}
$$

with

$$
\begin{aligned}
\omega= & -2 b d /\left(b^{2}+d^{2}\right)>0 \\
Q(\phi)= & x^{2}\left(\cos \phi-y z / x^{2}\right)^{2} \\
& +\left(x^{2}-y^{2}\right)\left(z^{2}-x^{2}\right) / x^{2} .
\end{aligned}
$$

Carrying out the integrations in (47), we obtain explicitly for $T \geq T_{c}(H)(z>y>x>0)$ the result [10]

$$
\begin{aligned}
& I_{1}= \frac{1}{2 \pi\left(z^{2}-x^{2}\right)^{1 / 2}} K(k), \\
& I_{2}= \frac{1}{2 \pi y z\left(z^{2}-x^{2}\right)^{1 / 2}}\left[z^{2} K(k)+\left(y^{2}-z^{2}\right) \Pi(r, k)\right] \\
& I_{3}=\frac{1}{2 \pi(y+\omega z)\left(z^{2}-x^{2}\right)^{1 / 2}}[y K(k) \\
&\left.\quad+\frac{\omega\left(z^{2}-y^{2}\right)}{z+\omega y} \Pi(s, k)\right]
\end{aligned}
$$

where

$$
\begin{aligned}
k^{2} & =\left(y^{2}-x^{2}\right) /\left(z^{2}-x^{2}\right), \\
r & =y^{2} / z^{2}, \\
s & =(\omega z+y)^{2} /(z+\omega y)^{2}
\end{aligned}
$$

and $K$ and $\Pi$ are elliptical integrals of the first and third kinds, respectively,

$$
\begin{aligned}
K(k) & =\int_{0}^{\pi / 2} \frac{d \alpha}{\sqrt{1-k^{2} \sin ^{2} \alpha}} \\
\Pi(r, k) & =\int_{0}^{\pi / 2} \frac{d \alpha}{\left(1-r \sin ^{2} \alpha\right) \sqrt{1-k^{2} \sin ^{2} \alpha}} .
\end{aligned}
$$

For $T \leq T_{c}(H)(y>z>x>0)$ we obtain the same result with $y$ and $z$ interchanged.

The apparent non-analyticity of $C_{0}$ at $\left|R_{1}\right|=|R|$ as indicated in (46) is spurious. It can be shown that the combination of $C_{0}+C_{3} I_{3}$ is always analytic.

At the transition temperature $y=z($ or $k=1, r=1)$ both $K$ and $\Pi$ diverge as $\ln \left|T-T_{c}(H)\right|$. Applying (45) to the internal energy (24) where the derivatives are with respect to $T$, we obtain

$$
\begin{array}{r}
U(H)=U_{c}(H)+\text { const }\left|T-T_{c}(H)\right| \ln \left|T-T_{c}(H)\right|, \\
T \rightarrow T_{c}(H) .
\end{array}
$$

A further derivative of $U(H)$ as given by (26) gives the specific heat $C_{H}$. Thus, the energy is continuous at $T_{c}(H)$ while the specific heat diverges logarithmically. These findings, which are the same as those found in the Fisher model, indicate the occurrence of a second order transition along the phase boundary in Fig. 3. We plot $U(H)$ and $C_{H}$ respectively in Figs. 4 and 5. For completeness, we derive the explicit expression for $U_{c}(H)$ in the next section.

In the case of zero magnetic field $H=\alpha=0$ and $\gamma=-1$, the internal energy assumes a simple expression given by

$$
\begin{aligned}
U(0) / J & =-1+(1+\tanh 2 K)\left\{1-\frac{1}{2} \tanh R\right. \\
- & {\left.\left[1+2\left(\cosh ^{3} 2 R-3 \cosh 2 R-2\right) I_{1}\right] \operatorname{coth} 2 R\right\}(53) }
\end{aligned}
$$

with $e^{-2 R}=e^{2 R_{1}}=\left(e^{4 K}+1\right) / 2$.

\section{DERIVATION OF $U_{c}(H)$}

Define an auxiliary variable

$$
\varphi_{\alpha}(k)=\sin ^{-1} \sqrt{\frac{1-\alpha}{1-k^{2}}}>0
$$




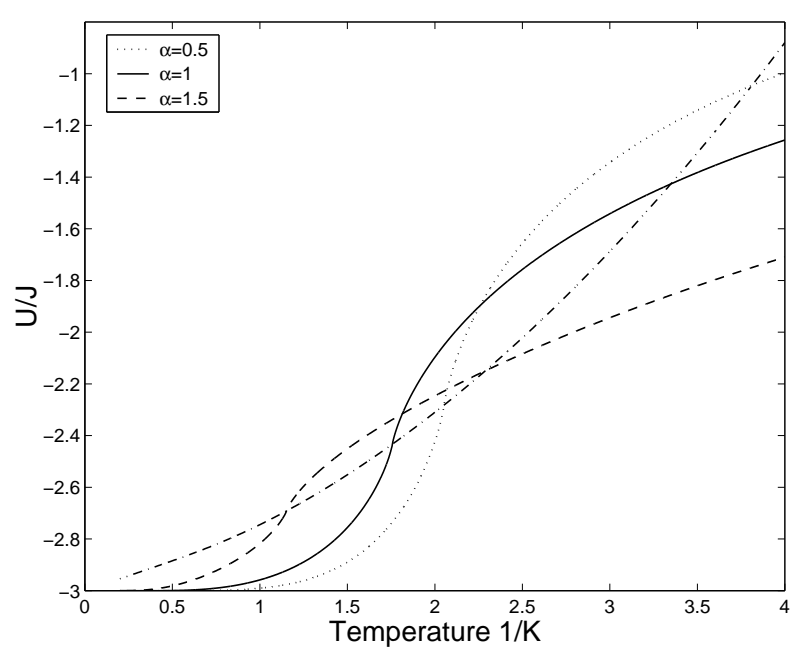

FIG. 4: Energy $U$ versus temperature $T$ at fixed magnetic field $\alpha$ for $\gamma=-1$. The broken line indicates the critical energy $U_{c}$.

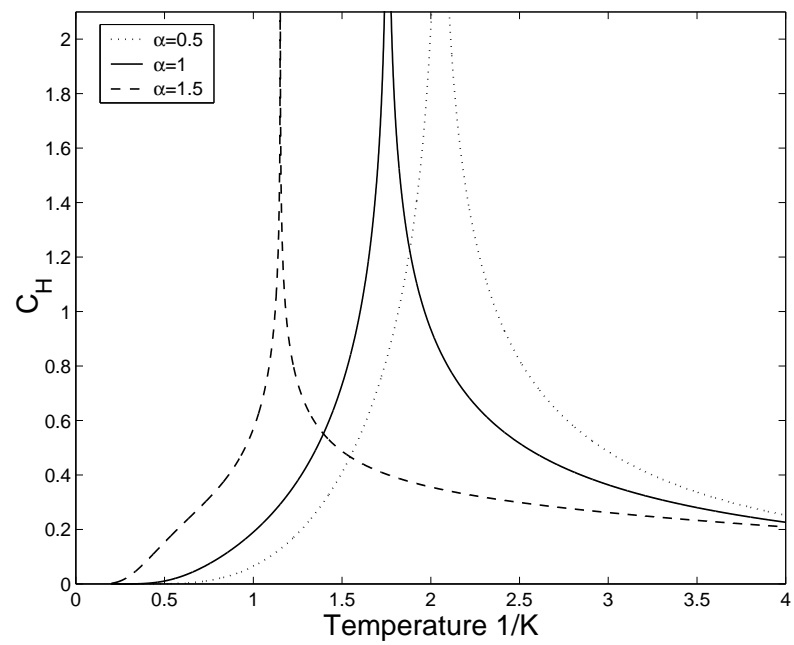

FIG. 5: Specific heat $C_{H}$ as a function of temperature $T$ at fixed magnetic field $\alpha$ for $\gamma=-1$.

which gives, for $k^{2}, r, s$ given in (50),

$$
\begin{aligned}
& \varphi_{r}(k)=\sin ^{-1} \sqrt{1-\frac{x^{2}}{z^{2}}} \\
& \varphi_{s}(k)=\sin ^{-1} \sqrt{\frac{\left(1-\omega^{2}\right)\left(z^{2}-x^{2}\right)}{(z+\omega y)^{2}}} \\
& \varphi_{s}(1)=\sin ^{-1} \sqrt{\frac{1-\omega}{1+\omega}\left(1-\frac{x^{2}}{z^{2}}\right)}, \quad T=T_{c}(H)
\end{aligned}
$$

where the last line holds at the critical temperature $k=1$ or $y=z$.

For $k^{2}<r<1$ we have [1]

$$
\Pi(r, k)=K(k)+\frac{\pi}{2} \sqrt{\frac{r}{(1-r)\left(r-k^{2}\right)}}\left[1-\Lambda_{0}\left(\varphi_{r}, k\right)\right]
$$

where $\Lambda_{0}$ is Heuman's Lambda function

$$
\Lambda_{0}(\varphi, k)=\frac{2}{\pi}\left[K(k) E\left(\varphi, k^{\prime}\right)-[K(k)-E(k)] F\left(\varphi, k^{\prime}\right)\right]
$$

with $k^{\prime}=\sqrt{1-k^{2}}$ and

$$
\begin{aligned}
& E(\varphi, k)=\int_{0}^{\varphi}\left(1-k^{2} \sin ^{2} \theta\right)^{1 / 2} d \theta \\
& F(\varphi, k)=\int_{0}^{\varphi}\left(1-k^{2} \sin ^{2} \theta\right)^{-1 / 2} d \theta .
\end{aligned}
$$

Particularly,

$$
\Lambda_{0}(\varphi, 1)=\frac{2}{\pi} \varphi
$$

At the critical point $k \rightarrow 1$ or $y \rightarrow z$, we have

$$
\begin{aligned}
& \left(z^{2}-y^{2}\right) \Pi(r, k)=\left(z^{2}-y^{2}\right) K(k) \\
& +\frac{\pi z^{2}\left(z^{2}-x^{2}\right)^{1 / 2}}{2 x}\left(1-\frac{2}{\pi} \varphi_{r}\right), \\
& \left(z^{2}-y^{2}\right) \Pi(s, k)=\left(z^{2}-y^{2}\right) K(k) \\
& \quad+\frac{\pi(1+\omega) z^{2}\left(z^{2}-x^{2}\right)^{1 / 2}}{2 \sqrt{(1-\omega)\left[(1-\omega) x^{2}-2 \omega z^{2}\right]}}\left(1-\frac{2}{\pi} \varphi_{s}\right),
\end{aligned}
$$

and

$$
\begin{aligned}
I_{2}= & I_{1}-\frac{1}{4 x}\left(1-\frac{2}{\pi} \varphi_{r}\right), \\
I_{3}= & \frac{1}{1+\omega}\left[I_{1}\right. \\
& \left.+\frac{\omega}{4 \sqrt{(1-\omega)\left[(1-\omega) x^{2}+2 \omega z^{2}\right]}}\left(1-\frac{2}{\pi} \varphi_{s}\right)\right] .
\end{aligned}
$$

Let

$$
q=\sinh ^{2} R_{1} \gtrless \frac{1}{2}, \quad\left|R_{1}\right| \gtrless|R| .
$$

Then, at the critical temperature $T_{c}(H)$

$$
\begin{aligned}
\omega= & \frac{4 q}{1+4 q^{2}}, \quad x=2(1+q) \\
y^{2}= & z^{2}=4(1+q)^{2}(1+2 q) / q \\
\varphi_{r}= & \sin ^{-1} \sqrt{\frac{1+q}{1+2 q}} \\
\varphi_{s}= & \sin ^{-1}\left[\frac{|2 q-1|}{2 q+1} \sqrt{\frac{1+q}{1+2 q}}\right] \\
I_{2}= & I_{1}-\frac{1}{8(1+q)}\left(1-\frac{2}{\pi} \varphi_{r}\right) \\
I_{3}= & \frac{1+4 q^{2}}{(1+2 q)^{2}}\left[I_{1}\right. \\
& \left.+\frac{q}{2(1+q)|1-2 q|(2 q+3)}\left(1-\frac{2}{\pi} \varphi_{s}\right)\right]
\end{aligned}
$$


and

$$
\begin{aligned}
C_{1}= & -\frac{(q+1)\left(4 q^{2}+8 q-3\right)}{2 q} \\
& \times\left(\operatorname{coth} 2 R_{1} \frac{\partial R_{1}}{\partial K}-\operatorname{coth} 2 R \frac{\partial R}{\partial K}\right) \\
C_{2}= & -2(1+q)\left(\operatorname{coth} 2 R_{1} \frac{\partial R_{1}}{\partial K}-\operatorname{coth} 2 R \frac{\partial R}{\partial K}\right) \\
C_{3}= & \frac{(q+1)(2 q-1)(2 q+3)(2 q+1)^{2}}{2 q\left(4 q^{2}+1\right)} \\
& \times\left(\operatorname{coth} 2 R_{1} \frac{\partial R_{1}}{\partial K}-\operatorname{coth} 2 R \frac{\partial R}{\partial K}\right)
\end{aligned}
$$

where

$$
\begin{aligned}
\frac{\partial R_{1}}{\partial K}= & \frac{2\left(e^{-4 \gamma K_{c}} \sinh 4 K_{c}-\gamma\right)}{e^{-4 \gamma K_{c}} \cosh 4 K_{c}-1}+\frac{2 \gamma}{e^{-4 \gamma K_{c}}-1} \\
\frac{\partial R}{\partial K}= & -\frac{e^{-4 \gamma K_{c}}\left(\sinh 4 K_{c}-2 \gamma \cosh ^{2} 2 K_{c}\right)+\alpha \sinh 4 \alpha K_{c}}{2\left(\sinh ^{2} 2 \alpha K_{c}+e^{-4 \gamma K_{c}} \cosh ^{2} 2 K_{c}\right)} \\
& +\alpha \tanh 2 \alpha K_{c}
\end{aligned}
$$

where $K_{c}=J / k T_{c}(H)$. Combining these results, we obtain after some algebra the following expression for the energy at the critical temperature:

$$
\begin{aligned}
& U_{c}(H) / J=-\gamma-2 \alpha \tanh 2 \alpha K_{c}-\frac{1}{2 \sinh 2 R_{1}} \frac{\partial R_{1}}{\partial K} \\
&+\frac{1}{2}(2-\operatorname{coth} 2 R) \frac{\partial R}{\partial K} \\
&+\frac{1}{2 \pi}\left(\varphi_{r} \pm \varphi_{s}\right)\left(\operatorname{coth} 2 R_{1} \frac{\partial R_{1}}{\partial K}-\operatorname{coth} 2 R \frac{\partial R}{\partial K}\right), \\
& q>\frac{1}{2} .
\end{aligned}
$$

In the limit of $\gamma \rightarrow 0$ for which $R_{1} \rightarrow \infty, \varphi_{r}=\varphi_{s}=$ $\pi / 4$, we obtain

$$
\begin{aligned}
U_{c}(H) /\left.J\right|_{\gamma=0} & =-(2-\sqrt{2}) \alpha \tanh 2 \alpha K_{c} \\
& -\frac{1}{2-\sqrt{2}} \frac{\sinh 4 K_{c}+\alpha \sinh 4 \alpha K_{c}}{\sinh ^{2} 2 \alpha K_{c}+\cosh ^{2} 2 K_{c}}
\end{aligned}
$$

which is the Fisher result [1]. Particularly, for $H=0$, (65) gives the value $-2 \sqrt{1+\sqrt{2}}$.

\section{MAGNETIC PROPERTIES}

For magnetic properties we take the derivative in (45) with respect to $H$ and obtain

$$
\begin{gathered}
M(H)=M_{c}(H)+\mathrm{const}\left|T-T_{c}(H)\right| \ln \left|T-T_{c}(H)\right|, \\
T \rightarrow T_{c}(H)
\end{gathered}
$$

where $M_{c}(H)$ is the magnetization at the critical temperature $T_{c}(H)$ given by

$$
\begin{aligned}
M_{c}(H)=\frac{1}{2}\left[1-\frac{1}{\pi}\left(\varphi_{r} \pm \varphi_{s}\right)\right] & \left(1+e^{4 R}\right) \tanh 2 \alpha K_{c}, \\
q & >\frac{1}{2}
\end{aligned}
$$

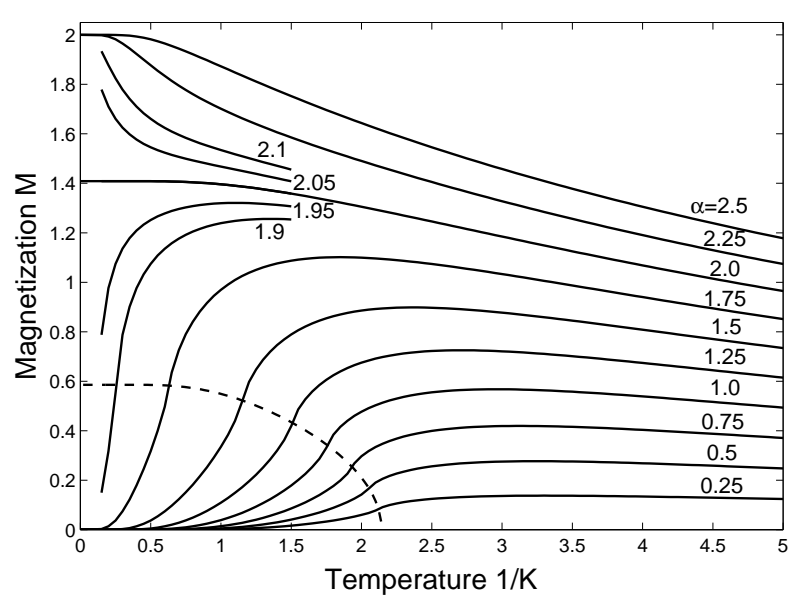

FIG. 6: Magnetization $M$ versus temperature $T$ at fixed magnetic field $\alpha$ for $\gamma=-1$. The broken line indicates $M_{c}(H)$.

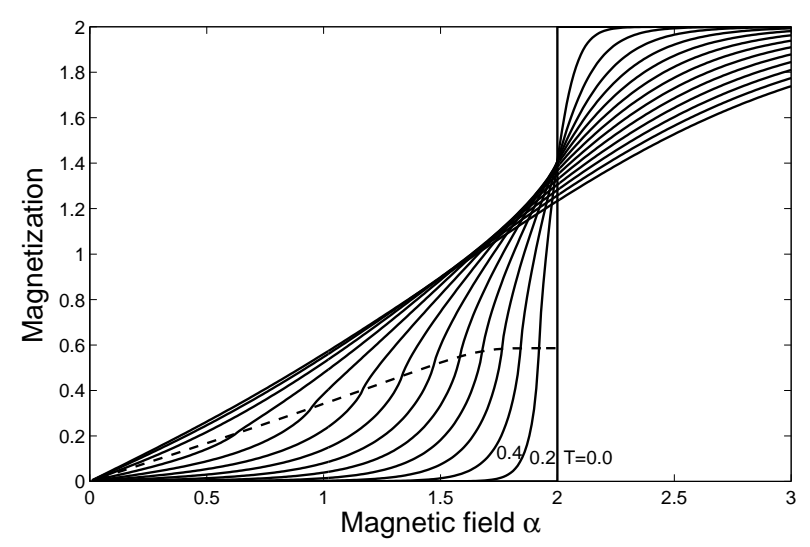

FIG. 7: Magnetization $M$ versus magnetic field $\alpha$ at fixed temperature $T$ for $\gamma=-1$. The broken line indicates $M_{c}(H)$.

The magnetization $M$ is plotted in Figs. 6 and 7 versus $T$ and $H$, respectively, for $\gamma=-1$. To analyze $M(H) \equiv$ $M(\gamma, \alpha, 1 / K)$ at low temperatures, we note that

$$
\begin{aligned}
M(\gamma, \alpha, 0) & =0, & & \alpha<1-\gamma \\
& =2, & & \alpha>1-\gamma .
\end{aligned}
$$

The value of $M(\gamma, \alpha, 0)$ at $\alpha=1-\gamma$ depends on how the zero temperature $T=0$ is approached. To see what happens we write

$$
\alpha(K, \xi) \equiv 1-\gamma-\frac{\xi}{4 K} \ln 2(\sqrt{2}+1)
$$

where $\xi$ is a parameter controlling the approach to $T=$ 0 . Particularly, the critical curve (34) is indicated by $\xi=1$. Along (69) and $K \rightarrow \infty$, one has $R_{1} \simeq 2 K \rightarrow \infty$, $\tanh L \rightarrow 1$, and

$$
e^{-4 R}=1+[2(\sqrt{2}+1)]^{\xi}, \quad K \rightarrow \infty .
$$


After some algebraic manipulation, we find

$$
\begin{aligned}
m(\xi) & \equiv \lim _{K \rightarrow \infty} M(\gamma, \alpha(K, \xi), 1 / K) \\
& =\left(e^{4 R}+1\right)\left[\frac{1}{2}+\frac{\epsilon}{\pi}(1-k) K(k)\right]
\end{aligned}
$$

with $k=\sinh ^{2 \epsilon} 2 R$. Here $\epsilon=1$ for $\xi \leq 1$ while $\epsilon=-1$ for $\xi>1$. We have

$$
\begin{aligned}
m(\xi) & =0 & & \xi=\infty \\
& =2-\sqrt{2} & & \xi=1 \\
& =\frac{3}{4}+\frac{21}{16 \pi} K\left(\frac{1}{8}\right) \simeq 1.408836 & & \xi=0 \\
& =2 & & \xi=-\infty .
\end{aligned}
$$

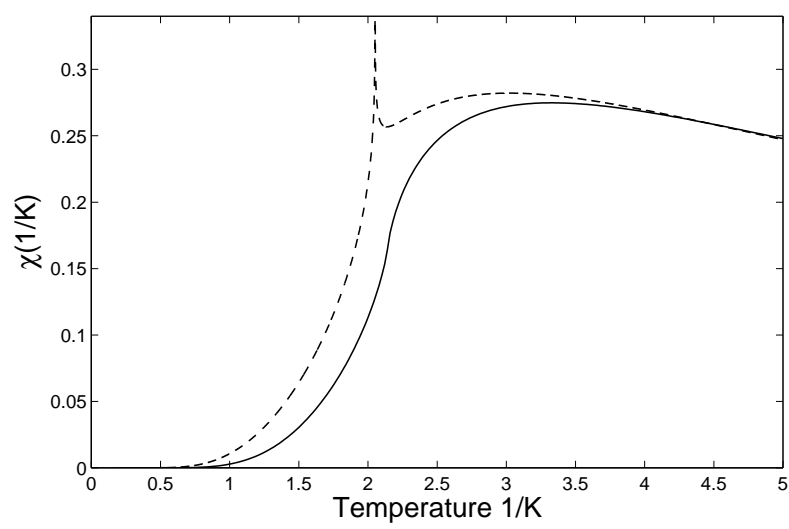

FIG. 8: Susceptibility as a function of temperature for $\gamma=$ -1 . Solid line $H=0$. Broken line $H=J(\alpha=1 / 2)$.
The susceptibility $\chi\left(\gamma, \alpha, K^{-1}\right)$, which is the further derivative of (66) with respect to $H$, diverges logarithmically at $T_{c}(H)$ for $H \neq 0$. The susceptibility $\chi$ is plotted in Fig. 8 versus temperature $T$ for $\gamma=-1$ and $\alpha=0,1 / 2$. The zero-field susceptibility $\chi\left(\gamma, 0, K^{-1}\right)$ is continuous. For example one has for $\gamma=-1$,

$$
\begin{aligned}
\chi\left(-1,0, K^{-1}\right) & =2 K-\frac{K}{2}\left(1-e^{4 R}\right)\left\{2-\frac{3}{2} \operatorname{coth} 2 R\right. \\
& +\operatorname{coth} 2 R\left[2 \cosh 2 R\left(3-\sinh ^{2} 2 R\right)+6\right] I_{1} \\
& \left.+\sinh 4 R I_{2}\right\}
\end{aligned}
$$

where $e^{-2 R}=\left(e^{4 K}+1\right) / 2$. The expression (72) bears no direct relation with the critical energy (53) as found to exist in the case of $\gamma=0$ [1]. By direct differentiation of (67) and making use of (37), we obtain the critical susceptibility

$$
\begin{aligned}
\chi\left(\gamma, 0, K_{c}^{-1}\right)= & \frac{1}{2} K_{c}\left[1-\frac{1}{\pi}\left(\varphi_{r} \pm \varphi_{s}\right)\right] \\
& \times\left(1+e^{4 \gamma K_{c}} \cosh ^{-2} 2 K_{c}\right), \quad q>\frac{1}{2}(73)
\end{aligned}
$$

\section{Acknowledgment}

We thank R. Shrock for his interest and a critical reading of the manuscript. Work has been supported in part by NSF Grants PHY-0098801 (WTL) and DMR-9980440 (FYW).
[1] M. E. Fisher, Proc. Roy. Soc. A 254, 66-85 (1960); ibid. 256 502-513 (1960).

[2] H. Giacomini, J. Phys. A 21, L31 (1988).

[3] K. Y. Lin, J. Phys. A 22, 3435 (1989).

[4] K. Obredors, A. Labarta, A. Isaigue, J. Tejeda, J. Rodrigues, and M. Pernet, Solid Stat. Commun. 65, 189-192 (1988).

[5] P. Azaria and H. Giacomini, J. Phys. A 21, L935 (1988).

[6] C. Fan and F. Y. Wu, Phys. Rev. B 2, 723-733 (1970).

[7] I. Syozi, Prog. Theor. Phys. 6, 306-308 (1951).

[8] I. Syozi, in Phase Transitions and Critical Phenomena Vol. 1, Eds. C. Domb and M. S. Green (Academic Press, New York 1972).
[9] R. M. F. Houtappel, Physica 16, 425-455 (1950).

[10] Expressions of $I_{1}, I_{2}, I_{3}$ given just above Eq. (41) in [ 6$]$ contain the following typos: The arguments $g$ of the elliptical integrals should read $q$. In the expression of $I_{1}$ replace $\left(z^{2}-x^{2}\right) \rightarrow\left(z^{2}-x^{2}\right)^{1 / 2}$, in the expression of $I_{2}$ multiply the whole expression by $1 / z$, and in the expression of $I_{3}$ replace the first $g$ by $y$.

[11] M. Abramowitz and I.A. Stegun, Handbook of mathematical functions with formulas, graphs, and mathematical tables (National Bureau of Standards, Applied Mathematics Series 55, 1970), Eqs. (17.7.14) and (17.4.40). 